\title{
Set-membership Identification of Block-Structured Nonlinear Feedback Systems
}

\author{
V. Cerone, D. Piga, D. Regruto
}

\begin{abstract}
In this paper a three-stage procedure for Setmembership identification of block-structured nonlinear feedback systems is proposed. Nonlinear block parameters bounds are computed in the first stage exploiting steady-state measurements. Then, given the uncertain description of the nonlinear block, bounds on the unmeasurable inner-signal are computed in the second stage. Finally, linear block parameters bounds are computed in the third stage on the basis of output measurements and computed inner signal bounds. Computation of both the nonlinear block parameters and the inner-signal bounds is formulated in terms of semialgebraic optimization and solved by means of suitable convex LMI relaxation techniques. Linear block parameters are bounded solving a number of linear programming problems.
\end{abstract}

Index Terms-bounded uncertainty, errors-in-variable, parameter bounding, LMI, linear programming.

\section{INTRODUCTION}

It is well known that extensive studies over the last decades in the identification of linear systems provided a well assessed methodology for the solution of modeling problems in the time or in the frequency domain through either recursive or batch scheme [1], [2] which, unfortunately, are not suitable for most real-life problems that are intrinsically nonlinear. A good number of effective techniques have been proposed in the literature for black-box identification of nonlinear systems: polynomial NARMAX models [3], Volterra and Wiener series expansions [4], Wavelets, Neural networks and Fuzzy Logic [5], Set-membership nonlinear identification [6] are only some examples. The main drawback, common to all such approaches, is the difficulty in exploiting prior information on the physical structure of the system to be identified. In order to overcome such a limitation, relevant efforts have been devoted in recent years to the problem of identifying the so called block structured nonlinear systems. Such a class of systems can be profitably used in order to obtain simple and effective models of a wide class of nonlinear systems through suitable interconnections of memoryless nonlinear gains and linear subsystems. Nonlinearities may enter the system in different ways: either at the input or at the output end or in the feedback path around a linear model. The configuration we are dealing with in this work, also referred to as a blockstructured nonlinear feedback system, is shown in Fig. 1; it consists of a feedback system with the linear dynamic model in the forward path and the static nonlinearity in the feedback path. It must be stressed that in this context only

The authors are with the Dipartimento di Automatica e Informatica, Politecnico di Torino, corso Duca degli Abruzzi 24, 10129 Torino, Italy; e-mail: vito.cerone@polito.it, dario.piga@polito.it, diego.regruto@polito.it; Tel: +39-011-564 7064; Fax: +39-011-564 7198 systems which intrinsecally show a nonlinear unaccessible feedback path are considered. This kind of model has been extensively studied in the context of the well known Lur'e problem [7], [8] for which a number of results are available as far as stability is concerned. The identification of such a model relies solely on the measurement of the input and the output signal of the whole system, $u_{t}$ and $y_{t}$ respectively, while all the internal signals are not assumed to be accessible.

A number of interesting applications of block-oriented feedback nonlinear models can be found in various engineering fields. This model has been successfully applied in [9] to describe the operation of dynamic mode atomic force microscopy; atomic force microscope cantilevers can be modeled as a feedback interconnection of a linear dynamic system and a nonlinear static function; the linear block describes the free cantilever dynamics while the feedback subsystem accounts for the sample interaction force, which is a nonlinear function of the tip-sample distance. In paper [10], Schoukens et. al study the identification of a block-structured nonlinear Wiener-Hammerstein system that is captured in the feedforward or the feedback path of a closed-loop system; the proposed method is illustrated on the identification of a microwave crystal detector. Ming-Tzu Ho and Jun-Ming Lu [11] consider the problem of synthesizing proportional-integral-derivative (PID) controllers for a given block-structured nonlinear feedback system in the presence of exogenous energy-bounded disturbance; their proposed synthesis method is used to design a controller for a ball and wheel system apparatus. In [12], Pearson and Pottmann describes a gray-box identification approach to three classes of block-oriented models: Hammerstein models, Wiener models, and the feedback block-oriented models and show an application of the proposed method to a simple first-principles model of a distillation columns. The rich diversity of hysteretic phenomena that can be generated by interconnecting a linear dynamic system with a feedback static nonlinearity, is investigated by $\mathrm{Oh}$ et al. in [13]. On the methodological side, further contributions to the identification of block-structured nonlinear feedback models can be found in [14], [15], [16] while the quite general class of block-structured systems which can be represented by a Linear Fractional Transformation (LFT) has recently been considered in [17], [18].

A common assumption in system identification is that the measurement error is statistically described. However, there are many cases where in practice random variable models are difficult to develop, while simple bounds are readily available. Some examples include mechanical tolerances, analog- 
to-digital converter quantization errors, systematic and class errors in measurement equipments. When uncertainties are assumed to belong to a given set, a set-membership characterization of measurement errors should be preferred to the stochastic description. In this context, all parameters consistent with the measurements, the error bounds and the assumed model structure, are feasible solutions of the identification problem. The interested reader can find further details on this approach in a number of survey papers (see, e.g., [19], [20]), in the book edited by Milanese et al. [21], and the special issues edited by Norton [22], [23].

In this work, we consider the identification of SISO discrete-time block-structured nonlinear feedback system when the nonlinear block can be modeled by a polynomial with finite and known order and the output measurement errors are bounded. Note that all the internal signals of the loop, that is $x_{t}$ and $\nu_{t}$, are not supposed to be measurable. To the authors' best knowledge, no contribution can be found in the literature on the identification problem addressed in this paper where we present a three-stage identification procedure.

The note is organized as follows. Section II is devoted to the formulation of the problem. In Section III, exploiting $M$ steady-state input-output data, uncertainty intervals on the parameters of the nonlinear static block are derived, solving two semialgebraic optimization problems for each parameter. Then, in Section IV, given the estimated uncertain nonlinearity $\mathcal{N}$ and the output measurements collected exciting the system with an input dynamic signal, bounds on each sample of the inner signal $x_{t}$ are computed solving two semialgebraic optimization problems. Both in the first and in the second stage suitable Linear Matrix Inequalities (LMI) relaxation techniques are exploited. Last, in Section $\mathrm{V}$, the bounds computed in the second stage, together with the output dynamic sequence, are used to obtain a polytopic outer approximation of the exact feasible parameter set of the linear system. A simulated example is reported in Section VI.

\section{PRoBlem FORMULATION}

Consider the SISO discrete-time block-structured nonlinear systems shown in Fig. 1, where the linear dynamic part is modeled by a discrete-time system which transforms $x_{t}$ into the noise-free output $w_{t}$ according to

$$
w_{t}=G\left(q^{-1}\right) x_{t}=\frac{B\left(q^{-1}\right)}{A\left(q^{-1}\right)} x_{t} .
$$

where $x_{t}=u_{t}-\nu_{t}$ is the unmeasurable inner signal and $A(\cdot)$ and $B(\cdot)$ are polynomials in the backward shift operator $q^{-1}$, $\left(q^{-1} w_{t}=w_{t-1}\right)$

$$
\begin{aligned}
& A\left(q^{-1}\right)=1+a_{1} q^{-1}+\ldots+a_{n a} q^{-n a}, \\
& B\left(q^{-1}\right)=b_{0}+b_{1} q^{-1}+\ldots+b_{n b} q^{-n b} .
\end{aligned}
$$

The nonlinear block transforms the noise-free output $w_{t}$ into $\nu_{t}$ according to

$$
\nu_{t}=\mathcal{N}\left(w_{t}, \gamma\right)=\sum_{k=1}^{n} \gamma_{k} w_{t}^{k}, \quad t=1, \ldots, N
$$

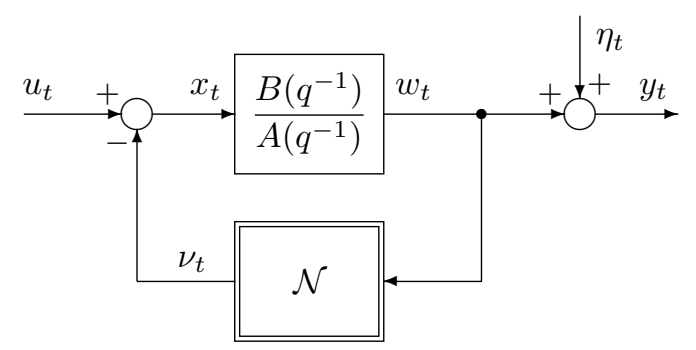

Fig. 1. Block-structured nonlinear feedback system.

where $n$ is the polynomial degree and $N$ is the length of the input sequence.

In line with the work done by a number of authors, it is assumed that: (i) $n$ is finite and a-priori known; (ii) both the linear part and the whole block-structured nonlinear feedback system are bounded input bounded output $\mathcal{L}_{p}$ stable; (iii) $\sum_{j=0}^{n b} b_{j} \neq 0$, that is, the steady-state gain of the linear block is not zero [24], [25]; (iv) an estimate of the process settling-time [26] is available. Let $y_{t}$ be the noise-corrupted measurements of $w_{t}$

$$
y_{t}=w_{t}+\eta_{t}
$$

Measurements uncertainty is known to be bounded by $\Delta \eta_{t}$, i.e.,

$$
\left|\eta_{t}\right| \leq \Delta \eta_{t}
$$

Unknown parameter vectors $\gamma \in R^{n}$ and $\theta \in R^{p}$ are defined, respectively, as

$$
\begin{aligned}
& \gamma^{T}=\left[\begin{array}{lll}
\gamma_{1} & \gamma_{2} & \ldots \gamma_{n}
\end{array}\right],
\end{aligned}
$$

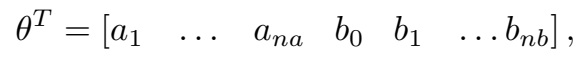

where $n_{a}+n_{b}+1=p$. It is easy to show that the parameterization of the structure of Fig. 1 is not unique. As a matter of fact, given a specific pair of subsystems $\tilde{G}\left(q^{-1}\right)$, $\tilde{\mathcal{N}}\left(w_{t}, \tilde{\gamma}\right)$, any dynamic system of the kind of the one depicted in Fig. 1 where $G\left(q^{-1}\right)=\tilde{G}\left(q^{-1}\right) /\left(1+\alpha \tilde{G}\left(q^{-1}\right)\right)$ and $\mathcal{N}\left(w_{t}, \tilde{\gamma}\right)=\tilde{\mathcal{N}}\left(w_{t}, \gamma\right)-\alpha w_{t}$, provides the same inputoutput behaviour for any constant $\alpha \in \Re$. Such an analysis is in perfect agreement with the detailed discussion provided in paper [15] where a structured Hammerstein-Nonlinear feedback model has been considered. In order to get a unique parameterization, in this work we assume, without loss of generality, that the steady-state gain $g$ of the linear block $G\left(q^{-1}\right)$ be 1 , that is:

$$
g=\frac{\sum_{j=0}^{n b} b_{j}}{1+\sum_{i=1}^{n a} a_{i}}=1
$$

In this paper we address the problem of deriving bounds on parameters $\gamma$ and $\theta$ consistently with given measurements, error bounds and the assumed model structure. The proposed solution is a three-stage procedure which will be described in the next sections. 


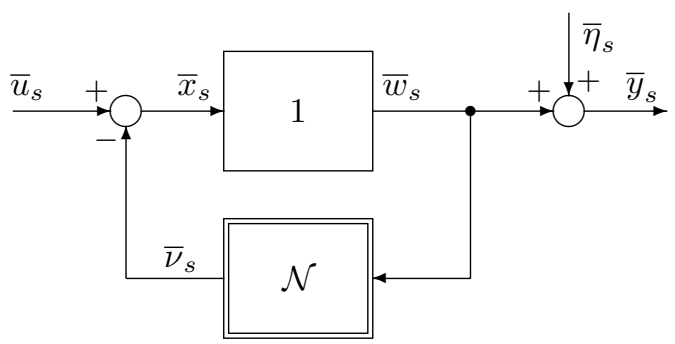

Fig. 2. Steady-state behaviour of the block-structured nonlinear feedback system.

\section{PARAMETERS BOUNDING OF THE NONLINEAR STATIC BLOCK}

Here we exploit steady-state operating conditions to bound the parameters of the nonlinear static block. Known input and noise corrupted output sequences are collected from the steady-state response of the system to a set of step inputs with different amplitudes. We only assume to have a rough idea of the system settling time, in order to know when steady-state conditions are reached, so that steady-state data can be collected. Indeed, under conditions (i), (ii) and (iii) stated in Section 2, combining equations (1), (4), (5), (6) and (9) in steady-state operating conditions we get the following equations describing the input-output mapping:

$$
\begin{aligned}
& \bar{w}_{s}+\sum_{k=1}^{n} \gamma_{k} \bar{w}_{s}^{k}-\bar{u}_{s}=0, \\
& \bar{w}_{s}=\bar{y}_{s}-\bar{\eta}_{s}, s=1, \ldots, M
\end{aligned}
$$

where $M \geq n$ is the number of measurement used in the identification of the nonlinear block, $\bar{u}^{\mathrm{T}}=\left[\bar{u}_{1}, \bar{u}_{2} \ldots \bar{u}_{M}\right]$ and $\bar{w}^{\mathrm{T}}=\left[\bar{w}_{1}, \bar{w}_{2} \ldots \bar{w}_{M}\right]$ are sequences of steady-state values of the known input signal and the unknown output signal respectively; $\bar{\eta}_{s}$ and $\bar{y}_{s}$ are the values of the noise and the measured output samples collected at some instant after the true system output has reached the steady-state value $\bar{w}_{s}$. Let us define $\bar{y}^{\mathrm{T}}=\left[\bar{y}_{1}, \bar{y}_{2} \ldots \bar{y}_{M}\right]$ and $\bar{\eta}^{\mathrm{T}}=\left[\bar{\eta}_{1}, \bar{\eta}_{2}, \ldots \bar{\eta}_{M}\right]$. A block diagram description of equations (10) is depicted in Fig. 2. The Feasible Parameter Set (FPS) of the static nonlinear block is defined as

$$
\begin{gathered}
\mathcal{D}_{\gamma}=\left\{\gamma \in R^{n}:\left(\bar{y}_{s}-\bar{\eta}_{s}\right)+\sum_{k=1}^{n} \gamma_{k}\left(\bar{y}_{s}-\bar{\eta}_{s}\right)^{k}=\bar{u}_{s},\right. \\
\left.\left|\bar{\eta}_{s}\right| \leq \Delta \bar{\eta}_{s} ; s=1, \ldots, M\right\},
\end{gathered}
$$

where $\Delta \bar{\eta}^{\mathrm{T}}=\left[\Delta \bar{\eta}_{1}, \Delta \bar{\eta}_{2}, \ldots \Delta \bar{\eta}_{M}\right]$ is the sequence of bounds on measurements uncertainty.

Definition (11) provides an exact, though implicit, description of the feasible parameter set of the nonlinear block which, due to the presence of the uncertain terms $\bar{\eta}_{s}$, $s=1, \ldots M$, is a non-convex region whose shape may become quite complex for increasing $n$ and $M$. Such an exact description of $\mathcal{D}_{\gamma}$ will be used here to compute the so called Parameter Uncertainty Intervals (PUI) of the nonlinear block parameters defined as:

$$
P U I_{\gamma_{j}}=\left\{\gamma_{j}^{\min }, \gamma_{j}^{\max }\right\}
$$

where

$$
\gamma_{j}^{\min }=\min _{\gamma \in \mathcal{D}_{\gamma}} \gamma_{j}, \quad \gamma_{j}^{\max }=\max _{\gamma \in \mathcal{D}_{\gamma}} \gamma_{j}
$$

The following result shows that the PUIs can be obtained solving two semi-algebraic optimization problems for each parameter $\gamma_{j}, j=1, \ldots n$.

Result 1 - Computation of $\gamma_{j}^{\min }$ and $\gamma_{j}^{\max }$ $\gamma_{j}^{\min }$ and $\gamma_{j}^{\max }$ can be computed solving the following optimization problems:

$$
\begin{aligned}
& \gamma_{j}^{\text {min }}=\min _{\gamma, \bar{\eta}} \gamma_{j} \\
& \text { s.t. } \\
& \qquad \begin{array}{l}
\left(\bar{y}_{s}-\bar{\eta}_{s}\right)+\sum_{k=1}^{n} \gamma_{k}\left(\bar{y}_{s}-\bar{\eta}_{s}\right)^{k}=\bar{u}_{s}, \\
\bar{\eta}_{s} \leq \Delta_{s}, \\
\bar{\eta}_{s} \geq-\Delta_{s}, \quad s=1, \ldots M
\end{array} \\
& \gamma_{j}^{\max }=\max _{\gamma, \bar{\eta}} \gamma_{j} \\
& \text { s.t. } \\
& \left\{\begin{array}{l}
\left(\bar{y}_{s}-\bar{\eta}_{s}\right)+\sum_{k=1}^{n} \gamma_{k}\left(\bar{y}_{s}-\bar{\eta}_{s}\right)^{k}=\bar{u}_{s}, \\
\bar{\eta}_{s} \leq \Delta_{s}, \\
\bar{\eta}_{s} \geq-\Delta_{s}, \quad s=1, \ldots M
\end{array}\right.
\end{aligned}
$$

Proof - Problems (14) and (15) are straightforwardly obtained from equations (13), assuming the nonlinear block parameters $\gamma_{j} j=1, \ldots n$ and the unknown noise samples $\bar{\eta}_{s} s=1, \ldots M$ as optimization variables.

Result 1 shows that computing tight bounds on each parameters $\gamma_{j}$ requires the computation of the global optima of two optimization problems, each consisting of the minimization (maximization) of a linear objective over a semi-algebraic set defined by $M$ multivariate polynomial equality constraints in the variables $\gamma, \bar{\eta}$ and $2 M$ linear inequality in the variable $\bar{\eta}$. Since such problems are non-convex, standard nonlinear optimization tools (gradient method, Newton method, etc.) cannot be used since they can trap in local minima which may result arbitrary far from the global one. As a consequence, the PUIs obtained using such tools are not guaranteed to contain the true unknown parameters, which is a key requirement of any set-membership identification method.

Considerable efforts have been devoted in recent years to the problem of computing approximate solutions to semialgebraic optimization problems by means of semidefinite relaxations techniques (see the survey paper [27] for a thorough review of the literature on the subject). Two alternatives and independently derived method have been proposed: the approach presented by Lasserre in [28] exploits the theory of moments while the one by Parrillo [29] is based on Sum Of Squares (SOS) optimization. In this work we exploit the approach proposed in [28]. The relaxation technique proposed by Lasserre is based 
on the idea of constructing an increasing sequence of convex LMI relaxations, whose optima are guaranteed to converge monotonically to the global optimum of the original nonconvex semialgebraic optimization problem. Although the method is guaranteed to converge as far as the length of the number of successive LMI relaxations (relaxation order) goes to infinity, exact global optima have been obtained for a number of small and medium size problems with a rather low relaxation order (see [28] for a collection of test problems solved with relaxation order less or equal than 4). An efficient MATLAB implementation of such a relaxation technique has been recently developed by Henrion and Lasserre in the open source freeware software Gloptipoly [30] which exploits the LMI solver SeDuMi [31] to solve semidefinite optimization problem in polynomial time. Application of the method proposed in [28] to problems (14) and (15) leads to the following results.

\section{Result 2 - Guaranteed relaxed parameter uncertainty intervals}

Let us call $\gamma_{j}^{\min ^{\delta}}$ and $\gamma_{j}^{\max ^{\delta}}$ the optimal solutions of the convex relaxations of order $\delta$ obtained by applying the Lasserre method to problems (14) and (15) respectively. The $\delta$-relaxed Parameter Uncertainty Interval $P U I_{\gamma_{j}}^{\delta}$ defined as:

$$
P U I_{\gamma_{j}}^{\delta}=\left[\gamma_{j}^{\min ^{\delta}}, \gamma_{j}^{\max ^{\delta}}\right], j=1 \ldots n
$$

is guaranteed to contain the true unknown parameter $\gamma_{j}$ to be estimated.

Proof - Statement of Result 2 follows from the definition of $\gamma_{j}^{\min }$ and $\gamma_{j}^{\max }$ provided by equation (13) and the fact that:

$$
\gamma_{j}^{\min ^{\delta}} \leq \gamma_{j}^{\min }, \quad \gamma_{j}^{\max ^{\delta}} \geq \gamma_{j}^{\max }, j=1 \ldots n
$$

Inequalities (17) are derived from the properties of method proposed by Lasserre.

\section{Result 3 - Convergence to tight parameter uncertainty intervals}

The $\delta$-relaxed parameter uncertainty interval $P U I_{\gamma_{j}}^{\delta}$ converges to the tight parameter uncertainty interval $P U I_{\gamma_{j}}$ as far as the relaxation order goes to infinity, i.e.:

$$
\begin{aligned}
& \lim _{\delta \rightarrow \infty} \gamma_{j}^{\min ^{\delta}}=\gamma_{j}^{\text {min }} \\
& \lim _{\delta \rightarrow \infty} \gamma_{j}^{\text {max }}=\gamma_{j}^{\text {max }}
\end{aligned}
$$

Proof - Statement of Result 3 follows directly from the convergence properties of Lasserre relaxation method.

As to the computational complexity of the proposed approach for the nonlinear block parameters bounds derivations, the two following results hold.

Result 4 - Complexity of relaxed bounds computation Computation of relaxed bounds $\gamma_{j}^{\min ^{\delta}}$ and $\gamma_{j}^{\max \delta}$ through direct application of the Lasserre method to problems (14) and (15) requires the solutions of two convex LMI optimization problems where:

4.1 The number of LMI decision variables and the size of the LMI grow polynomially in the relaxation order $\delta$.

4.2 The number of LMI decision variables and the size of the LMI grow polynomially in the measurements number $M$.

Proof - Fact 4.1 is directly obtained from the analysis of complexity of the Lasserre method with respect to the relaxation order $\delta$. Statement 4.2 comes from the following two considerations:

- the number of LMI variables and the size of the LMI in the Lasserre method grows polynomially in the number of polynomial variables of the original nonconvex semialgebraic problem;

- the number of polynomial variables in the semialgebraic problems (14) and (15) grows linearly in the number of measurement $M$.

The complexity analysis presented in Result 4 has been obtained from direct application of the Lasserre method to problems (14) and (15). The next result shows that, exploiting the peculiar structure of the considered problem, the complexity of the proposed bounding algorithm can be reduced through a proper reformulation of the LMI relaxation in the spirit of the work of Waki et al. [32]

\section{Result 5 - Reduced complexity computation of relaxed bounds}

Exploiting the fact that each optimization variable $\bar{\eta}_{s}, s=$ $1, \ldots M$ enters only one of the polynomial constraints of problem (14) and (15), a suitable reformulation of the LMI relaxation can be obtained where:

5.1 The number of LMI decision variables and the size of the LMI grow polynomially in the order of relaxation $\delta$.

5.2 The number of LMI decision variables and the size of the LMI grow linearly in the number of measurements $M$.

Due to the limited number of available pages, Result 5 is not proved in the paper; its proof can be found in [33].

\section{BOUNDING THE UNMEASURABLE INNER SIGNAL $x_{t}$}

In the second stage of our procedure we stimulate the block-structured nonlinear feedback system of Fig. 1 with a persistently exciting input signal $u_{t}$ and evaluate bounds on each sample of the corresponding unmeasurable inner signal $x_{t}$. For each input sequence sample $u_{t}$, bounds on the corresponding inner unmeasurable signal sample $x_{t}$ can be evaluated through the following expressions

$$
x_{t}^{\min }=u_{t}-\nu_{t}^{\max }, \quad x_{t}^{\max }=u_{t}-\nu_{t}^{\min }
$$

where

$$
\nu_{t}^{\max }=\max _{\gamma \in \mathcal{D}_{\gamma},\left|\eta_{t}\right| \leq \Delta \eta_{t}} \varphi_{t}^{\mathrm{T}} \gamma
$$


and

$$
\nu_{t}^{\min }=\min _{\gamma \in \mathcal{D}_{\gamma},\left|\eta_{t}\right| \leq \Delta \eta_{t}} \varphi_{t}^{\mathrm{T}} \gamma
$$

where $\varphi_{t}^{\mathrm{T}}=\left[\begin{array}{lll}\left(y_{t}-\eta_{t}\right) & \left(y_{t}-\eta_{t}\right)^{2} & \left(y_{t}-\eta_{t}\right)^{3} \ldots\left(y_{t}-\eta_{t}\right)^{n}\end{array}\right]$. The following result shows that bounds $\nu_{t}^{\max }$ and $\nu_{t}^{\min }$ on the sample $\nu_{t}$ can be computed solving two semi-algebraic optimization problems.

\section{Result 6 - Computation of $\nu_{t}^{\min }$ and $\nu_{t}^{\max }$}

$\nu_{t}^{\min }$ and $\nu_{t}^{\max }$ can be computed solving the following optimization problems:

$$
\begin{aligned}
\nu_{t}^{\min } & =\min _{\gamma, \bar{\eta}, \eta_{t}} \varphi_{t}^{\mathrm{T}} \gamma \\
& \text { s.t. } \\
& \left\{\begin{array}{l}
\left(\bar{y}_{s}-\bar{\eta}_{s}\right)+\sum_{k=2}^{n} \gamma_{k}\left(\bar{y}_{s}-\bar{\eta}_{s}\right)^{k}=\bar{u}_{s}, \\
\bar{\eta}_{s} \leq \Delta_{s}, \bar{\eta}_{s} \geq-\Delta_{s}, \quad s=1, \ldots M \\
\eta_{t} \leq \Delta \eta_{t}, \eta_{t} \geq-\Delta \eta_{t}
\end{array}\right. \\
\nu_{t}^{\max } & =\max _{\gamma, \bar{\eta}, \eta_{t}} \varphi_{t}^{\mathrm{T}} \gamma \\
& \text { s.t. } \\
& \left\{\begin{array}{l}
\left(\bar{y}_{s}-\bar{\eta}_{s}\right)+\sum_{k=2}^{n} \gamma_{k}\left(\bar{y}_{s}-\bar{\eta}_{s}\right)^{k}=\bar{u}_{s}, \\
\bar{\eta}_{s} \leq \Delta_{s}, \bar{\eta}_{s} \geq-\Delta s \\
\eta_{t} \leq \Delta \eta_{t}, \eta_{t} \geq-\Delta \eta_{t}
\end{array} \quad \begin{array}{l}
s, \ldots M
\end{array}\right.
\end{aligned}
$$

Result 6 can be proved on the basis of the same reasoning presented in the proof of Result 1 .

Problems (23) and (24) are addressed in this paper through the same relaxation method exploited in Section III for the nonlinear block parameters bounds computation. As a consequence, the properties presented in Results 2, 3, 4 and 5 also hold as far as the computation of bounds on $\nu_{t}$ is concerned.

\section{BOUNDING THE PARAMETERS OF THE LINEAR DYNAMIC MODEL}

Given bounds $x_{t}^{\min }$ and $x_{t}^{\max }$ computed in the second stage of the procedure, a compact description of the inner unmeasurable signal $x_{t}$ in terms of its central value $x_{t}^{c}$ and its perturbation $\delta x_{t}$ is given by:

$$
x_{t}=x_{t}^{c}+\delta x_{t}
$$

where

$$
\begin{gathered}
\left|\delta x_{t}\right| \leq \Delta x_{t} . \\
x_{t}^{c}=\frac{x_{t}^{\text {min }}+x_{t}^{\text {max }}}{2} \\
\Delta x_{t}=\frac{x_{t}^{\text {max }}-x_{t}^{\text {min }}}{2}
\end{gathered}
$$

Thanks to such a description of the unknown signal $x_{t}$, we can formulate the identification of the linear model in terms of the noisy output sequence $\left\{y_{t}\right\}$ and the uncertain inner sequence $\left\{x_{t}\right\}$ as shown in Fig. 3. Such a formulation is commonly referred to as an errors-in-variables problem (EIV), i.e. a parameter estimation problem in a linear-inparameter model where the output and some or all the

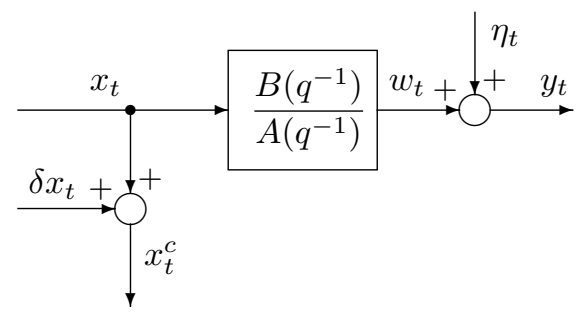

Fig. 3. Errors-in-variables set-up for bounding the parameters of the linear system.

explanatory variables are uncertain. As a matter of fact, combining equations (1), (2), (3), (5), (25) we get

$y_{t}=-\sum_{i=1}^{n a}\left(y_{t-i}-\eta_{t-i}\right) a_{i}+\sum_{j=0}^{n b}\left(x_{t-j}^{c}+\delta x_{t-j}\right) b_{j}+\eta_{t}$.

The linear system feasible parameter region is defined as

$$
\begin{gathered}
\mathcal{D}_{\theta}=\left\{\theta \in R^{p}: A\left(q^{-1}\right)\left[y_{t}-\eta_{t}\right]=B\left(q^{-1}\right)\left[x_{t}^{c}+\delta x_{t}\right] ;\right. \\
\left.g=1 ;\left|\eta_{t}\right| \leq \Delta \eta_{t} ;\left|\delta x_{t}\right| \leq \Delta x_{t} ; t=1, \ldots, N\right\} .
\end{gathered}
$$

From equation (29) it can be seen that consecutive regressions are related deterministically by uncertain output samples and uncertain input samples (dynamic EIV) giving rise to possible nonlinear exact parameter bounds, which could be not easily and exactly computed [34]. On the other end, when the uncertain variables appearing in successive regressions are supposed to vary independently (static EIV) exact parameter bounds are piecewise linear and, thus, as shown in [34], can be more conveniently handled than the FPS of dynamic EIV. That motivates the use, in this paper, of results from the static EIV [35] which, however, will lead to outer approximations of the exact FPS. Thus, in this work, a polytopic outer approximation $\mathcal{D}_{\theta}^{\prime}$ of the exact FPS $\mathcal{D}_{\theta}$, i.e. $\mathcal{D}_{\theta}^{\prime} \supset \mathcal{D}_{\theta}$, will be presented, together with an orthotopeouter bounding set $\mathcal{B}_{\theta}$ of $\mathcal{D}_{\theta}^{\prime}$. When we apply results from [35] to our problem we get the following description of the feasible parameter set $\mathcal{D}_{\theta}^{\prime}$ at the single time $t$

$$
\begin{aligned}
& \left(\phi_{t}-\Delta \phi_{t}\right)^{\mathrm{T}} \theta \leq y_{t}+\Delta \eta_{t} \\
& \left(\phi_{t}+\Delta \phi_{t}\right)^{\mathrm{T}} \theta \geq y_{t}-\Delta \eta_{t}
\end{aligned}
$$

where

$$
\begin{gathered}
\phi_{t}^{\mathrm{T}}=\left[\begin{array}{llll}
-y_{t-1} \ldots-y_{t-n a} & x_{t}^{c} x_{t-1}^{c} \ldots x_{t-n b}^{c}
\end{array}\right] \\
\Delta \phi_{t}^{\mathrm{T}}=\left[\begin{array}{llll}
\Delta \eta_{t-1} \operatorname{sgn}\left(a_{1}\right) & \ldots & \Delta \eta_{t-n a} \operatorname{sgn}\left(a_{n a}\right) \\
\Delta x_{t} \operatorname{sgn}\left(b_{0}\right) & \Delta x_{t-1} \operatorname{sgn}\left(b_{1}\right) & \ldots & \Delta x_{t-n b} \operatorname{sgn}\left(b_{n b}\right)
\end{array}\right]
\end{gathered}
$$

The orthotope-outer bounding set $\mathcal{B}_{\theta}$ is defined as

$$
\begin{array}{r}
\mathcal{B}_{\theta}=\left\{\theta \in R^{p}: \theta_{j}=\theta_{j}^{c}+\delta \theta_{j},\right. \\
\left.\left|\delta \theta_{j}\right| \leq \Delta \theta_{j}, j=1, \ldots, p\right\},
\end{array}
$$

where

$$
\begin{gathered}
\theta_{j}^{c}=\frac{\theta_{j}^{\text {min }}+\theta_{j}^{\text {max }}}{2}, \\
\Delta \theta_{j}=\frac{\theta_{j}^{\text {max }}-\theta_{j}^{\text {min }}}{2},
\end{gathered}
$$


and

$$
\theta_{j}^{\min }=\min _{\theta \in \mathcal{D}_{\theta}^{\prime}} \theta_{j}, \quad \theta_{j}^{\max }=\max _{\theta \in \mathcal{D}_{\theta}^{\prime}} \theta_{j}
$$

Parameter vectors $\gamma^{c}$ and $\theta^{c}$ are Chebishev centers in the $\ell_{\infty}$ norm of $\mathcal{D}_{\gamma}$ and $\mathcal{D}_{\theta}^{\prime}$ respectively and are commonly referred to as central estimates.

\section{A SimUlATED EXAMPLE}

In this section we illustrate the proposed parameter bounding procedure through a numerical example. The system considered here is characterized by (2), (3) and (4) with: $\gamma_{1}=-1.5, \gamma_{2}=1.2, \gamma_{3}=0.9, \quad A\left(q^{-1}\right)=(1-$ $\left.1.5193 q^{-1}+0.5326 q^{-2}\right)$ and $B\left(q^{-1}\right)=\left(0.1549 q^{-1}-\right.$ $\left.0.1416 q^{-2}\right)$. Thus, the true parameter vectors are $\gamma^{\mathrm{T}}=$ $\left[\begin{array}{lll}\gamma_{1} & \gamma_{2} & \gamma_{3}\end{array}\right]=\left[\begin{array}{lll}-1.5 & 1.2 & 0.9\end{array}\right]$ and $\theta^{\mathrm{T}}=\left[\begin{array}{llll}a_{1} & a_{2} & b_{1} & b_{2}\end{array}\right]=$ $\left[\begin{array}{llll}-1.5193 & 0.5326 & 0.1549 & -0.1416\end{array}\right]$. From the simulated transient sequence $\left\{w_{t}, \eta_{t}\right\}$ and steady-state data $\left\{\bar{w}_{s}, \bar{\eta}_{s}\right\}$, the signal to noise ratios $S N R$ and $\overline{S N R}$ are evaluated, respectively, through

$$
\begin{aligned}
& S N R=10 \log \left\{\sum_{t=1}^{N} w_{t}^{2} / \sum_{t=1}^{N} \eta_{t}^{2}\right\} \\
& \overline{S N R}=10 \log \left\{\sum_{s=1}^{M} \bar{w}_{s}^{2} / \sum_{s=1}^{M} \bar{\eta}_{s}^{2}\right\}
\end{aligned}
$$

Bounded absolute output error has been considered for simulating the collection of both steady state data, $\left\{\bar{u}_{s}, \bar{y}_{s}\right\}$, and transient sequence $\left\{u_{t}, y_{t}\right\}$. Here we assumed $\left|\eta_{t}\right| \leq \Delta \eta_{t}$ and $\left|\bar{\eta}_{s}\right| \leq \Delta \bar{\eta}_{s}$ where $\eta_{t}$ and $\bar{\eta}_{s}$, are random sequences belonging to the uniform distributions $U\left[-\Delta \eta_{t},+\Delta \eta_{t}\right]$ and $U\left[-\Delta \bar{\eta}_{s},+\Delta \bar{\eta}_{s}\right]$ respectively. Bounds on steady-state and transient output measurement errors were supposed to have the same value, i.e., $\Delta \eta_{t}=\Delta \bar{\eta}_{s} \triangleq \Delta \eta$, and were chosen in such a way as to have a signal to noise ratio output between $20 \mathrm{db}$ and $30 \mathrm{db}$. For a given $\Delta \eta$, the length of steady-state and the transient data are $M=50$ and $N=[100,300]$ respectively. The steady-state input sequence $\left\{\bar{u}_{s}\right\}$ belongs to the interval $[-10,+10]$, while the transient input sequence $\left\{u_{t}\right\}$ belongs to the uniform distribution $U[-10,+10]$. The signal to noise ratio $\overline{S N R}$ in the steady-state sequence is $28 \mathrm{db}$, while the signal to noise ratio SNR in the transient sequence is $24 \mathrm{db}$ and $25 \mathrm{db}$ when $N=100$ and $N=300$ respectively. Results about the nonlinear block are reported in Table I, where it is possible to see the parameter central estimates $\left(\gamma_{j}^{c}\right)$, the parameters bounds $\left(\gamma_{j}^{\min }, \gamma_{j}^{\max }\right)$ and the parameter uncertainty bounds $\Delta \gamma_{j}$ defined as:

$$
\Delta \gamma_{j}=\frac{\gamma_{j}^{\max }-\gamma_{j}^{\min }}{2}
$$

obtained for a LMI relaxation order $\delta=3$. Table II shows the numerical results obtained for the linear system, including parameter central estimates $\left(\theta_{j}^{c}\right)$, parameters bounds $\left(\theta_{j}^{\text {min }}\right.$, $\theta_{j}^{\max }$ ) and parameter uncertainty bounds $\Delta \theta_{j}$ defined in (37). As the number of transient observations increases (from $N=100$ to $N=300$ ), the width of all parameter uncertainty bounds of the linear system $\Delta \theta_{j}$ decreases, as expected.
Inner signal bounds, have been obtained solving the LMI relaxation problem with $\delta=3$.

\section{CONCLUSIONS}

A three-stage procedure has been presented for parameters bounds computation of block-structured nonlinear feedback systems. The algorithm proposed in the first stage for the computation of nonlinear block parameter bounds is based on the solution of a suitable nonconvex optimization problem by means of LMI relaxations techniques. The parameter uncertainty intervals computed solving the relaxed problems are proved to contain the unknown parameters to be estimated. Besides, such parameter bounds are proved to converge to the tight ones defined as the solution of the original nonconvex problems, as the relaxation order goes to infinity. Analogous results also hold for the algorithm proposed in the second stage for the computation of bounds on the unmeasurable inner signal. The problem of bounding the linear block parameter is formulated in terms of ErrorsIn-Variables identification and solved by means of linear programming. The proposed technique requires to perform two different specific experiments, a steady-state one and a transient one. Thus, in general, it cannot be applied when only one data set is available unless both steady-state and transient data can be separately extracted from such a set.

Table I: Nonlinear block parameter central estimates $\left(\gamma_{j}^{c}\right)$, parameter bounds $\left(\gamma_{j}^{\min }, \gamma_{j}^{\max }\right)$ and parameter uncertainty bounds $\left(\Delta \gamma_{j}\right)$ against true values.

\begin{tabular}{ccccc}
\hline $\begin{array}{c}\text { True } \\
\text { Value }\end{array}$ & $\gamma_{j}^{\text {min }}$ & $\gamma_{j}^{c}$ & $\gamma_{j}^{\max }$ & $\Delta \gamma_{j}$ \\
\hline & & & & \\
\hline-1.5000 & -1.5369 & -1.4890 & -1.4410 & 0.0480 \\
1.2000 & 1.1931 & 1.2072 & 1.2213 & 0.0141 \\
0.9000 & 0.8898 & 0.9020 & 0.9141 & 0.0121 \\
\hline
\end{tabular}

Table II: Linear system parameter central estimates $\left(\theta_{j}^{c}\right)$, parameter bounds $\left(\theta_{j}^{\min }, \theta_{j}^{\max }\right)$ and parameter uncertainty bounds $\left(\Delta \theta_{j}\right)$ against true values and number of transient samples $(N)$.

\begin{tabular}{rrrrrr}
\hline $\mathrm{N}$ & $\begin{array}{r}\text { True } \\
\text { Value }\end{array}$ & $\theta_{j}^{\text {min }}$ & $\theta_{j}^{c}$ & $\theta_{j}^{\text {max }}$ & $\Delta \theta_{j}$ \\
& & & & & \\
\hline \multirow{2}{*}{100} & -1.5193 & -2.0326 & -1.6422 & -1.2518 & 0.3904 \\
& 0.5326 & 0.3046 & 0.6364 & 0.9681 & 0.3318 \\
& 0.1549 & 0.1424 & 0.1579 & 0.1734 & 0.0155 \\
& -0.1416 & -0.2201 & -0.1232 & -0.0264 & 0.0969 \\
\hline \multirow{3}{*}{300} & & & & & \\
& -1.5193 & -1.8569 & -1.5633 & -1.2697 & 0.2936 \\
& 0.5326 & 0.3265 & 0.5761 & 0.8256 & 0.2496 \\
& 0.1549 & 0.1452 & 0.1555 & 0.1659 & 0.0104 \\
& -0.1416 & -0.1951 & -0.1348 & -0.0746 & 0.0602 \\
\hline
\end{tabular}




\section{REFERENCES}

[1] L. Ljung, System Identification, Theory for the User. Upper Saddle River: Prentince Hall, 1999.

[2] T. Söderström and P. Stoica, System Identification. Upper Saddle River: Prentice Hall, 1989.

[3] I. J. Leonaritis and S. A. Billings, "Input-output parametric models for nonlinear systems," International Journal of Control, vol. 41, pp. 303-344, 1985.

[4] W. Rugh, Nonlinear System Theory: The Volterra/Wiener Approach. Baltimore: Johns Hopkins Univ. Press, 1981.

[5] J. Sjöberg, Q. Zhang, L. Ljung, A. Benveniste, B. Delyon, P. Glorennec, H. Hjalmarsson, and A. Juditsky, "Nonlinear black-box modeling in system identification: a unified overview," Automatica, vol. 31, no. 12, pp. 1691-1724, 1995.

[6] C. Novara and M. Milanese, "Set-membership identification of nonlinear systems," Automatica, vol. 40, no. 6, pp. 957-975, 2004.

[7] M. Vidyasagar, Nonlinear Systems Analysis. Englewood Cliffs, NJ: Prentice Hall, 1978.

[8] H. K. Khalil, Nonlinear Systems. Upper Saddle River, NJ: Prentice Hall, 2000

[9] M. Basso, D. Materassi, and M. Salapaka, "Hysteresis models of dynamic mode atomic force microscopes: analysis and identification via harmonic balance," Nonlinear Dynamics, vol. 54, pp. 297-306, 2008.

[10] J. Schoukens, L. Gommé, W. V. Moer, and Y. Rolain, "Identification of a block-structured nonlinear feedback system, applied to a microwave crystal detector," IEEE Trans. Instrumentation and Measurement, vol. 57, no. 8, pp. 1734-1740, 2008.

[11] M.-T. Ho and J.-M. Lu, " $H_{\infty}$ PID controller design for Lur'e systems and its application to a ball and wheel apparatus," Int. J. of Control, vol. 78, no. 1, pp. 53-64, 2005.

[12] R. K. Pearson and M. Pottmann, "Gray-box identification of blockoriented nonlinear models," J. of Process Control, vol. 10, pp. 301315, 2000.

[13] J. Oh, B. Drincic, and D. S. Bernstein, "Nonlinear feedback models of hysteresis," IEEE Control Systems Magazine, vol. 29, no. 1, pp. 100-119, 2009.

[14] S. A. Billings and S. Y. Fakhouri, "Identification of systems containing linear dynamic and static nonlinear elements," Automatica, vol. 18, no. 1 , pp. $15-26,1982$.

[15] T. H. V. Pelt and D. S. Bernstein, "Nonlinear system identification using Hammerstein and nonlinear feedback models with piecewise linear static map," Int. J. of Control, vol. 74, no. 18, pp. 1807-1823, 2001.

[16] L. Lauwers, J. Schoukens, R. Pintelon, and M. Enqvist, "A nonlinear block structure identification procedure using frequency response function measurement," IEEE Trans. Instrumentation and Measurement, vol. 57, no. 10, pp. 2257-2264, 2008.

[17] K. Hsu, K. Poolla, and T. Vincent, "Identification of structured nonlinear systems," IEEE Transaction on Automatic Control, vol. 53, no. 11, pp. 2497-2513, 2008.

[18] E. Pepona, S. Paoletti, A. Garulli, and P. Date, "An iterative piecewise affine identification of nonlinear interconnected systems," in Proc. of the 46th IEEE Conference on Decision and Control, 2007, pp. 50985103.

[19] M. Milanese and A. Vicino, "Optimal estimation theory for dynamic sistems with set membership uncertainty: an overview," Automatica, vol. 27(6), pp. 997-1009, 1991.

[20] E. Walter and H. Piet-Lahanier, "Estimation of parameter bounds from bounded-error data: a survey," Mathematics and Computers in simulation, vol. 32, pp. 449-468, 1990.

[21] M. Milanese, J. Norton, H. Piet-Lahanier, and E. Walter, Eds., Bounding approaches to system identification. New York: Plenum Press, 1996.

[22] J. Norton (Ed.), "Special issue on bounded-error estimation," Int. J. of Adapt. Control \& Sign. Proces., vol. 8, no. 1, 1994.

[23] — "Special issue on bounded-error estimation," Int. J. of Adapt. Control \& Sign. Proces., vol. 9, no. 1, 1995.

[24] Z. Lang, "Controller design oriented model identification method for Hammerstein system," Automatica, vol. 29, no. 3, pp. 767-771, 1993.

[25] L. Sun, W. Liu, and A. Sano, "Identification of a dynamical system with input nonlinearity," IEE Proc. Part D, vol. 146, no. 1, pp. 41-51, 1999.
[26] A. Kalafatis, L. Wang, and W. Cluett, "Identification of Wiener-type nonlinear systems in a noisy enviroment," Int. J. Control, vol. 66, no. 6, pp. 923-941, 1997.

[27] M. Laurent, "Sums of squares, moment matrices and optimization over polynomials," Emerging Applications of Algebraic Geometry, Vol. 149 of IMA Volumes in Mathematics and its Applications, M. Putinar and S. Sullivant (eds.), pp. 157-270, 2009

[28] J. B. Lasserre, "Global optimization with polynomials and the problem of moments," SIAM Journal on Optimization, vol. 11, pp. 796-817, 2001

[29] P. Parrillo, "Semidefinite programming relaxations for semialgebraic problems," Mathematical Programming, vol. 96, pp. 293-320, 2003.

[30] D. Henrion and J. B. Lasserre, "Gloptipoly: Global optimization over polynomials with Matlab and SeDuMi," ACM Transactions Math. Soft, vol. 29, pp. 165-194, 2003.

[31] J. F. Sturm, "Using SeDuMi 1.02, a MATLAB Toolbox for optimization over symmetric cones," Optim. Methods Software, vol. 11, no. 12, pp. 625-653, 1999.

[32] H. Waki, S. Kim, M. Kojima, and M. Muramatsu, "Sums of squares and semidefinite programming relaxations for polynomial optimization problems with structured sparsity," SIAM Journal on Optimization, vol. 17, no. 1, pp. 218-242, 2006.

[33] V. Cerone, D. Piga, and D. Regruto, "Parameters bounds of blockstructured nonlinear feedback systems," DAUIN Internal Report 2009/02/07, 2009.

[34] S. Veres and J. Norton, "Parameter-bounding algorithms for linear errors in variables models," in Proc. of IFAC/IFORS Symposium on Identification and System Parameter Estimation, 1991, pp. 1038-1043.

[35] V. Cerone, "Feasible parameter set for linear models with bounded errors in all variable," Automatica, vol. 29, no. 6, pp. 1551-1555, 1993 